\title{
A statistical parsimony method for uncertainty quantification of FDTD computation based on the PCA and ridge regression
}

DOI:

10.1109/tap.2019.2911645

\section{Document Version}

Accepted author manuscript

Link to publication record in Manchester Research Explorer

Citation for published version (APA):

Hu, R., Monebhurrun, V., Himeno, R., Yokota, H., \& Costen, F. (2019). A statistical parsimony method for uncertainty quantification of FDTD computation based on the PCA and ridge regression. IEEE Transactions on Antennas and Propagation . https://doi.org/10.1109/tap.2019.2911645

\section{Published in:}

IEEE Transactions on Antennas and Propagation

\section{Citing this paper}

Please note that where the full-text provided on Manchester Research Explorer is the Author Accepted Manuscript or Proof version this may differ from the final Published version. If citing, it is advised that you check and use the publisher's definitive version.

\section{General rights}

Copyright and moral rights for the publications made accessible in the Research Explorer are retained by the authors and/or other copyright owners and it is a condition of accessing publications that users recognise and abide by the legal requirements associated with these rights.

\section{Takedown policy}

If you believe that this document breaches copyright please refer to the University of Manchester's Takedown Procedures [http://man.ac.uk/04Y6Bo] or contact uml.scholarlycommunications@manchester.ac.uk providing relevant details, so we can investigate your claim.

\section{OPEN ACCESS}




\title{
A statistical parsimony method for uncertainty quantification of FDTD computation based on the PCA and ridge regression
}

\author{
Runze Hu, Vikass Monebhurrun, Senior Member, IEEE, Ryutaro Himeno, Hideo Yokota, Fumie Costen, Senior \\ Member, IEEE
}

\begin{abstract}
The non-intrusive polynomial chaos (NIPC) expansion method is one of the most frequently used methods for uncertainty quantification (UQ) due to its high computational efficiency and accuracy. However, the number of polynomial bases is known to substantially grow as the number of random parameters increases, leading to excessive computational cost. Various sparse schemes such as the least angle regression method have been utilised to alleviate such a problem. Nevertheless, the computational cost associated with the NIPC method is still nonnegligible in systems which consist of a high number of random parameters. This paper proposes the first versatile UQ method which requires the least computational cost whilst maintaining the UQ accuracy. We combine the hyperbolic scheme with the principal component analysis method and reduce the number of polynomial bases with the simpler procedure than currently available, keeping most information in the system. The ridge regression method is utilised to build a statistical parsimonious model to decrease the number of input samples and the leaveone-out cross-validation method is applied to improve the UQ accuracy .
\end{abstract}

Index Terms-Non-intrusive polynomial chaos expansion (NIPC), principal component analysis (PCA), ridge regression (RR), uncertainty quantification, finite difference time domain (FDTD), Debye media

\section{INTRODUCTION}

An abstract of a physical phenomenon can be ideally represented by a physical model consisting of a set of equations, enabling scientists to elucidate the laws of physics such as optics and electromagnetism. The modeling technique [1] has become an essential tool over recent decades, being propelled by advances in computer simulation in the design of manufactured products and the numerical predictions of experimental observations.

The finite difference time domain (FDTD) [2], [3] method is a powerful and robust technique to model inhomogeneous

R. $\mathrm{Hu}$ and F. Costen are with the School of Electrical and Electronic Engineering, The University of Manchester, U.K. (email: fumie.costen@manchester.ac.uk).

V. Monebhurrun is with the EXPOSE/PIEM/GEEPS, CentraleSupelec, 11 rue Joliot Curie Plateau de Moulon, Gif sur Yvette 91192, France (e-mail: Vikass.MONEBHURRUN@centralesupelec.fr).

R. Himeno is with Head Office for Information Systems and Cybersecurity, RIKEN, Saitama, Japan.

H. Yokota and F. Costen are with the Image Processing Research Team, Centre for Advanced Photonics, RIKEN, Saitama, Japan.

Color version of the figures in this paper are available online at http://ieeexplore.ieee.org.

Additional research data supporting this publication are available from http://dx.doi.org/ repository at doi: 10.17632/8484k6yh2p.1 materials, making it ideally suited for bioelectromagnetic simulations. This paper deals with the numerical simulation of the propagation of electromagnetic waves in the human body. In the FDTD calculations, the one-pole Debye model [4] is utilised to describe the frequency-dependent behaviour of human tissues whose frequency response varies from person to person. Such variation is termed as uncertainty.

This paper focuses on quantifying the uncertainty of the FDTD results produced by the propagation of uncertainty of input Debye-parameters. The methods utilised for uncertainty quantification (UQ) can be classified as either intrusive or nonintrusive. The intrusive UQ methods, such as the stochastic Galerkin scheme [5], require the modification of existing deterministic code, which take risks of introducing errors to the well-validated simulators. On the other hand, non-intrusive UQ methods [6]-[8], such as the Monte Carlo method (MCM) [9][11], stochastic collocation based method [12]-[14] and nonintrusive polynomial chaos (NIPC) expansion method [15][17] treat the simulator as a black box without changing the underlying simulator code.

Although the MCM is considered a gold standard technique for UQ, it requires a large number of simulations to produce satisfactory results, leading to computational inefficiency. The NIPC expansion method offers an ideal alternative to MCM. It estimates outputs of the system using a series of orthogonal polynomials. In NIPC, the input parameters are expanded with a certain number of polynomial chaos (PC) bases, and the projection method [18] or the regression method [13], [19] can be utilised to estimate the coefficients of these PC bases. In the projection method, the coefficients in NIPC can be estimated by the quadrature rule or MCM, while the regression method is based on the least square minimisation of the error between the system output and its approximation. However, both methods are prone to the curse of dimensionality [20], whereby the number of required simulations grows substantially as the number of input parameters increases.

\section{A. Related work}

Many advanced UQ techniques have been proposed over the last decade to lift the curse of dimensionality. Ideas behind these techniques include sparse strategies, the hierarchical approach and model order reduction.

In general, higher-order PC bases are usually less important than the lower-order PC bases and so preservation of the latter 
is usually prioritised when it comes to the selection of PC bases. The work in [21] builds the sparse PC expansion using the q-quasi-norm [22], [23]. Such norms penalise the highorder PC bases, resulting in only significant bases retained in the PC expansion. The techniques based on the analysis of variance (ANOVA) [24]-[29] have also been utilised to exploit the sparsity of the generalised PC expansion. For instance, [27] develops a hierarchical stochastic spectral simulator based on the anchored ANOVA in which an anchor point and a threshold are introduced to the ANOVA in order to obtain a truncated ANOVA decomposition. [30] proposes an ANOVAbased stochastic simulator to efficiently simulate subsystems within a hierarchical system. This technique treats the outputs of subsystem as new random inputs of the entire system, thereby considerably reducing the number of input parameters.

[31] utilises the adaptive $L_{0}$ regularisation to perform the feature selection in order to ascertain a unique solution for an underdetermined equation. [32] proposes a method based on stochastic collocation to solve the high-dimensional UQ problems, whereby a tensor recovery approach is adopted to significantly reduce the cost of tensor product. [33] proposes a model reduction technique to obtain the optimum number of samples based on the quadrature point sampling method [34]. Such reduced models can preserve the important properties of the original system. Furthermore, most UQ techniques assume that the input parameters are Gaussian and correlated. The work in [35] generalises the stochastic collocation method to quantify the uncertainty of systems which consist of nonGaussian and correlated input parameters.

\section{B. Paper contributions}

This paper extends a recently developed least angle regression [21], [36]-[40] based UQ method [41] aiming to further reduce the computational cost of UQ for FDTD computation. Our proposed method consists of three stages; sample selection, sparsity process and UQ.

- Sample selection: An efficient sampling method is crucial for determining the accuracy of UQ results. This paper adopts the Latin hypercube sampling (LHS) method [42], [43] for sample selection, making use of its property of variance reduction and its capability to preserve the real variability of Debye-parameters within selected samples.

- Sparsity process: This stage combines the principal component analysis (PCA) [44], [45] method with the hyperbolic scheme [23] to minimise the number of PC bases and improve the stability of UQ results. The PCA method transforms a large set of input parameters into a smaller set while retaining a high proportion of the information from the original set of data. Such a procedure reduces the number of input parameters. The transformed input parameters are then utilised to construct the sparse PC expansion based on the hyperbolic scheme. As a consequence, only a quarter of the PC bases are required, in comparison to the number utilised for NIPC expansion via general truncation [20].

- Uncertainty quantification: We adopt the ridge regression (RR) method [46], [47] to estimate the coefficients of
PC bases. Following the sparsity process, the number of input samples may fall below the number of PC bases leading to the NIPC expansion being underdetermined. In this case, the ordinary least square (OLS) method [48] is unable to provide a unique solution. However, the RR method is capable of handling such a problem. It introduces a penalty term to the regression method and makes the matrix inversion function irrespective of the number of input samples without degrading the accuracy of UQ results.

Furthermore, the RR method can deal with the problem of multicollinearity which exists among PC bases [49]. In statistics, multicollinearity is a phenomenon in which PC bases correlate with one another, with the influence of each PC basis on the UQ results hard to isolate and distinguish [50], [41]. The coefficient estimation for these correlated PC bases is therefore unreliable.

Due to the ease of implementation and its non-parametric nature [51], PCA has gained high popularity in its ability to help determine UQ over the past decade [52]-[56]. Various extensions of PCA, such as kernel PCA [57] and non-linear PCA [58], have been proposed to solve specific problems. This paper adopts standard PCA for dimensionality reduction in order to reduce the complexity of the entire UQ algorithm. Although standard PCA has some limitations [51], the influence of these limitations on UQ results is trivial. For example, one of the limitations of PCA is that the transformed principle components are difficult to interpret. However, such a limitation does not impact the non-intrusive UQ method.

This paper is organised as follows: Section II explains the principle of the hyperbolic scheme, the PCA method, the RR method and the proposed method. Section III details the numerical experiments for UQ by the MCM and the proposed method. Finally, we discuss the results from these techniques with the merits of the proposed method outlined and evaluated.

\section{Methodology}

The principles of the hyperbolic scheme, the PCA method, the RR method and the proposed method are presented in Section III The hyperbolic scheme enables us to build a sparse PC expansion. The PCA method is capable of reducing a number of input parameters thereby further reducing the number of PC bases. The RR method and the proposed method are used for UQ based on the sparse PC expansion.

\section{A. The hyperbolic scheme for PC expansion}

The PC basis is defined as $\psi_{b_{1} b_{2} \cdots b_{\mathcal{K}}}\left(\xi_{1}, \xi_{2}, \cdots, \xi_{\mathcal{K}}\right)$, where $b_{1} b_{2} \cdots b_{\mathcal{K}}$ is the index of the PC basis, and $\xi_{1}, \xi_{2}, \cdots, \xi_{\mathcal{K}}$ are $\mathcal{K}$ input parameters. In this paper, each input parameter $\xi_{k}$ represents a certain Debye parameter of a certain tissue and the electric field $\boldsymbol{E}$ is observed in the FDTD simulations. The highest order of the PC basis in a PC expansion is denoted as $r \in[1,+\infty)$. We set $r$ to a certain finite value so that the PC expansion is truncated from the infinite PC expansion. In this case, $b_{k}$ satisfies 


$$
\sum_{k=1}^{\mathcal{K}} b_{k} \leq r, b_{k} \geq 0
$$

and the total number of PC bases in the PC expansion is

$$
\mathcal{L}=\frac{(\mathcal{K}+r) !}{\mathcal{K} ! r !} .
$$

The combination of $b_{1} b_{2} \ldots b_{\mathcal{K}}$ can be restricted to satisfy

$$
\left(\sum_{k=1}^{\mathcal{K}} b_{k}^{q}\right)^{\frac{1}{q}} \leq r
$$

for $0<q \leq 1$, and thus the number of PC bases in the PC expansion can be reduced from (2) based on $q$-quasi-norm. The details of the hyperbolic scheme is explained in [41].

\section{B. The principal component analysis}

PCA is a dimension-reduction method used to transform a set of parameters into a number of uncorrelated parameters, namely, principal components. The PCA starts with the standardisation of the data. We define a matrix $\mathcal{X}=\left(\xi_{k}{ }^{(m)}, m=\right.$ $1 \sim \mathcal{M}, k=1 \sim \mathcal{K})$ as a sample set which consists of $\mathcal{M} \boldsymbol{\xi}^{(m)}$, where the vector $\boldsymbol{\xi}$ is a combination of $\mathcal{K}$ Debye parameters of interest as in $\boldsymbol{\xi}=\left[\xi_{1}, \xi_{2}, \ldots, \xi_{\mathcal{K}}\right]$ and $\boldsymbol{\xi}^{(m)}$ indicates the $m$-th $\boldsymbol{\xi} . \mathcal{X}$ is presented as

$$
\boldsymbol{\mathcal { X }}=\left[\begin{array}{cccc}
\xi_{1}{ }^{(1)} & \xi_{2}{ }^{(1)} & \ldots & \xi_{\mathcal{K}}{ }^{(1)} \\
\xi_{1}{ }^{(2)} & \xi_{2}{ }^{(2)} & \ldots & \xi_{\mathcal{K}}{ }^{(2)} \\
\vdots & \vdots & \ddots & \vdots \\
\xi_{1}{ }^{(\mathcal{M})} & \xi_{2}{ }^{(\mathcal{M})} & \ldots & \xi_{\mathcal{K}}^{(\mathcal{M})}
\end{array}\right]=\left[\begin{array}{c}
\boldsymbol{\xi}^{(1)} \\
\boldsymbol{\xi}^{(2)} \\
\vdots \\
\boldsymbol{\xi}^{(\mathcal{M})}
\end{array}\right]
$$

Let $\boldsymbol{\xi}_{k}$ be a vector consisting of $\mathcal{M}$ samples for $\xi_{k}$ as in $\boldsymbol{\xi}_{k}=\left[\xi_{k}{ }^{(1)}, \xi_{k}{ }^{(2)}, \ldots, \xi_{k}{ }^{(\mathcal{M})}\right]^{T}$, and matrix $\widetilde{\mathcal{X}}$ be the standardised $\mathcal{X}$ whose elements are $\tilde{\xi}_{k}^{(m)}=\frac{\xi_{k}{ }^{(m)}-\mu_{k}}{\sigma_{k}}$, where $\mu_{k}$ is the average value of $\boldsymbol{\xi}_{k}$ for $\mathcal{M}$ samples calculated as in $\mu_{k}=\frac{1}{\mathcal{M}} \sum_{m=1}^{\mathcal{M}} \xi_{k}{ }^{(m)}$, and $\sigma_{k}$ is the standard deviation of $\boldsymbol{\xi}_{k}$ for $\mathcal{M}$ samples calculated as in $\sigma_{k}^{2}=$ $\frac{1}{\mathcal{M}-1} \sum_{m=1}^{\mathcal{M}}\left(\xi_{k}{ }^{(m)}-\mu_{k}\right)^{2}$. The standardisation process enables all the $\tilde{\xi}$ in $\tilde{\mathcal{X}}$ to have the same measurement scale [59] thereby improving the accuracy of PCA.

Let $\tilde{\xi}_{k}=\left[\tilde{\xi}_{k}^{(1)}, \tilde{\xi}_{k}^{(2)}, \ldots, \tilde{\xi}_{k}^{(\mathcal{M})}\right]^{T}$ be a vector consisting of $\mathcal{M}$ samples for $\tilde{\xi}_{k}$. We construct the covariance matrix $\mathcal{G}=\left(\mathcal{G}_{i j}, i=1 \sim \mathcal{K}, j=1 \sim \mathcal{K}\right)$ where $\mathcal{G}_{i j}$ is the covariance between $\tilde{\xi}_{i}$ and $\tilde{\xi}_{j}$ calculated by $\mathcal{G}_{i j}=\frac{1}{\mathcal{M}-1} \sum_{m=1}^{\mathcal{M}}\left(\tilde{\xi}_{i}{ }^{(m)}-\right.$ $\left.\frac{1}{\mathcal{M}} \sum_{m^{\dagger}=1}^{\mathcal{M}} \tilde{\xi}_{i}^{\left(m^{\dagger}\right)}\right)\left(\tilde{\xi}_{j}{ }^{(m)}-\frac{1}{\mathcal{M}} \sum_{m^{\dagger}=1}^{\mathcal{M}} \tilde{\xi}_{j}^{\left(m^{\dagger}\right)}\right) \cdot \mathcal{G}$ is then decomposed into eigenvectors and eigenvalues as in

$$
\mathcal{G}=\mathcal{P} \mathcal{H} \mathcal{P}^{-1},
$$

where $\mathcal{P}$ is a $\mathcal{K} \times \mathcal{K}$ matrix which is the combination of a set of $\mathcal{K}$ eigenvectors and $\mathcal{H}$ is the eigenvalue matrix presented as $\mathcal{H}=\left[\begin{array}{cccc}\mathcal{H}_{1} & 0 & \ldots & 0 \\ 0 & \mathcal{H}_{2} & \ldots & 0 \\ \vdots & \vdots & \ddots & \vdots \\ 0 & 0 & \ldots & \mathcal{H}_{\mathcal{K}}\end{array}\right]$

The $\mathcal{K}$ diagonal elements of $\mathcal{H}$ as in $\operatorname{diag}(\mathcal{H})=$ $\left\{\mathcal{H}_{1}, \mathcal{H}_{2}, \ldots, \mathcal{H}_{\mathcal{K}}\right\}$ are the eigenvalues of $\mathcal{G}$. The eigenvalues are also called the characteristic roots used as the explanatory importance with respect to $\xi_{k}$.

The eigenvectors indicate the principal components of $\mathcal{G}$. We define the vector $\widetilde{\mathcal{H}}=\left\{\widetilde{\mathcal{H}}_{1}, \widetilde{\mathcal{H}}_{2}, \ldots, \widetilde{\mathcal{H}}_{\mathcal{K}}\right\}$ as the numerically sorted $\operatorname{diag}(\mathcal{H})$ in descending order of $\widetilde{\mathcal{H}}_{1} \geq \widetilde{\mathcal{H}}_{2} \ldots \geq$ $\widetilde{\mathcal{H}}_{\mathcal{K}}$ and $\mathcal{C}=\left\{\mathcal{C}_{1}, \mathcal{C}_{2}, \ldots, \mathcal{C}_{\mathcal{K}}\right\}$ as a vector of variance explained as calculated in

$$
\mathcal{C}_{k}=\frac{\widetilde{\mathcal{H}}_{k}}{\sum_{k=1}^{\mathcal{K}} \widetilde{\mathcal{H}}_{k}}
$$

The vector of the cumulative variance explained $\widehat{\mathcal{C}}=$ $\left\{\widehat{\mathcal{C}}_{1}, \widehat{\mathcal{C}}_{1}, \ldots, \widehat{\mathcal{C}}_{\mathcal{K}}\right\}$ is the cumulative summation of the elements of $\mathcal{C}$ given by

$$
\widehat{\mathcal{C}_{k}}=\sum_{i=1}^{k} \mathcal{C}_{i}, k=1 \sim \mathcal{K} .
$$

$\widehat{\mathcal{C}}$ indicates how much information the first $\mathcal{K}$ eigenvectors carry. In PCA, we select the first $\mathcal{D}$ principal components $(\mathcal{D} \leq \mathcal{K})$ which satisfies $\widehat{\mathcal{C}_{\mathcal{D}}} \geq(1-\rho)$, where $\rho$ is a certain percentage of the information to be dropped.

Let $\mathcal{P}$ be the $\mathcal{K} \times \mathcal{D}$ matrix of eigenvectors when $\mathcal{D}$ principal components are kept. The original sample set is then transformed into a new subset as in

$$
\mathcal{X}_{\text {sub }}=\tilde{\mathcal{X}} \widehat{\mathcal{P}}=\left[\hat{\boldsymbol{\xi}}^{(1)}, \hat{\boldsymbol{\xi}}^{(2)}, \cdots, \hat{\boldsymbol{\xi}}^{(\mathcal{M})}\right]^{T},
$$

where vector $\hat{\boldsymbol{\xi}}^{(m)}=\left[\hat{\xi}_{1}{ }^{(m)}, \hat{\xi}_{2}{ }^{(m)}, \ldots, \hat{\xi}_{\mathcal{D}}{ }^{(m)}\right]$ is the combination of the $\mathcal{D}$ Debye parameters in $\boldsymbol{\mathcal { X }}_{\text {sub }}$ and $\hat{\boldsymbol{\xi}}^{(m)}$ indicates the $m$-th $\hat{\xi}$. The $\mathcal{K}$-dimensional feature space is therefore transformed into a $\mathcal{D}$-dimensional feature subspace.

\section{The ridge regression method}

In the NIPC method, the multicollinearlity exists among the PC bases. For example, when considering 3 input parameters, the Hermite polynomials $\psi_{100}\left(\xi_{1}, \xi_{2}, \xi_{3}\right)=$ $\xi_{1}$ and $\psi_{200}\left(\xi_{1}, \xi_{2}, \xi_{3}\right)=\xi_{1}^{2}-1$. The relationship of $\psi_{100}\left(\xi_{1}, \xi_{2}, \xi_{3}\right)$ and $\psi_{200}\left(\xi_{1}, \xi_{2}, \xi_{3}\right)$ can be written as $\psi_{200}\left(\xi_{1}, \xi_{2}, \xi_{3}\right)=\psi_{100}\left(\xi_{1}, \xi_{2}, \xi_{3}\right)^{2}-1$, meaning $\psi_{100}\left(\xi_{1}, \xi_{2}, \xi_{3}\right)$ and $\psi_{200}\left(\xi_{1}, \xi_{2}, \xi_{3}\right)$ are in perfect correlation because the value of $\psi_{200}\left(\xi_{1}, \xi_{2}, \xi_{3}\right)$ is completely dependent on the value of $\psi_{100}\left(\xi_{1}, \xi_{2}, \xi_{3}\right)$. As a consequence, the NIPC method is unable to accurately distinguish the influence of $\psi_{100}\left(\xi_{1}, \xi_{2}, \xi_{3}\right)$ on the UQ results and thereby the estimated coefficient of $\psi_{100}\left(\xi_{1}, \xi_{2}, \xi_{3}\right)$ is unreliable.

In the projection method, the input samples to the system are predetermined among the vast number of random samples 
and the number of input parameters cannot be reduced in a computationally-efficient manner [60], leading to the high computational cost. On the other hand, the regression method can vary the number of input samples. Hence, this paper focuses on the regression method to estimate the coefficients of PC bases. The regression method performs the matrix inversion under the condition [19] that the number of input samples exceeds the number of PC bases, i.e. oversampling. Such a condition restricts the minimum number of input samples. Relatively high number of input samples may hinder the computational efficiency of the NIPC method. However, this does not necessarily mean that the regression method is unable to provide an optimal solution for UQ when this condition is not met.

The RR method has the potential to analyse the data that suffers from multicollinearity, and it also enables the NIPC method to function irrespective of the number of samples.

In the RR method, the NIPC expansion is expressed as a linear regression model of

$$
\mathcal{T}\left(\boldsymbol{\xi}^{(m)}\right)=\sum_{l=1}^{\mathcal{L}} a_{\boldsymbol{b}_{l}} \psi_{\boldsymbol{b}_{l}}\left(\boldsymbol{\xi}^{(m)}\right),
$$

where $\mathcal{T}\left(\boldsymbol{\xi}^{(m)}\right)$ is the predicted value of $|\boldsymbol{E}|^{2}$ for $\boldsymbol{\xi}^{(m)}$, the polynomial term $a_{\boldsymbol{b}_{l}} \psi_{\boldsymbol{b}_{l}}\left(\boldsymbol{\xi}^{(m)}\right)$ is called a predictor of $\mathcal{T}\left(\boldsymbol{\xi}^{(m)}\right), \boldsymbol{b}_{l}$ indicates the index of the $l$-th $\psi$, and $a$ is the coefficient of $\psi$.

We define $\boldsymbol{a}$ as a vector consisting of $\mathcal{L}$ coefficients as in $\boldsymbol{a}=\left[a_{\boldsymbol{b}_{1}}, \cdots, a_{\boldsymbol{b}_{\mathcal{L}}}\right]^{T}$, and the PC bases matrix $\boldsymbol{\Psi}=$ $\left(\psi_{\boldsymbol{b}_{l}}\left(\boldsymbol{\xi}^{(m)}\right), m=1 \sim \mathcal{M}, l=1 \sim \mathcal{L}\right)$ is presented as $\boldsymbol{\Psi}=\left[\begin{array}{cccc}\psi_{\boldsymbol{b}_{1}}\left(\boldsymbol{\xi}^{(1)}\right) & \psi_{\boldsymbol{b}_{2}}\left(\boldsymbol{\xi}^{(1)}\right) & \ldots & \psi_{\boldsymbol{b}_{\mathcal{L}}}\left(\boldsymbol{\xi}^{(1)}\right) \\ \psi_{\boldsymbol{b}_{1}}\left(\boldsymbol{\xi}^{(2)}\right) & \psi_{\boldsymbol{b}_{2}}\left(\boldsymbol{\xi}^{(2)}\right) & \ldots & \psi_{\boldsymbol{b}_{\mathcal{L}}}\left(\boldsymbol{\xi}^{(2)}\right) \\ \vdots & \vdots & \ddots & \vdots \\ \psi_{\boldsymbol{b}_{1}}\left(\boldsymbol{\xi}^{(\mathcal{M})}\right) & \psi_{\boldsymbol{b}_{2}}\left(\boldsymbol{\xi}^{(\mathcal{M})}\right) & \ldots & \psi_{\boldsymbol{b}_{\mathcal{L}}}\left(\boldsymbol{\xi}^{(\mathcal{M})}\right)\end{array}\right]$.

$\boldsymbol{a}$ is calculated by

$$
\boldsymbol{a}=\left(\boldsymbol{\Psi}^{T} \boldsymbol{\Psi}+v \boldsymbol{1}\right)^{-1} \boldsymbol{\Psi}^{T} \mathcal{E},
$$

where $v$ is a constant named as penalty factor,

$$
\mathcal{E}=\left[\left|\boldsymbol{E}_{1}\right|^{2}, \cdots,\left|\boldsymbol{E}_{\mathcal{M}}\right|^{2}\right]^{T}
$$

is a vector of the $\mathcal{M}|\boldsymbol{E}|^{2}$ obtained by the FDTD simulation, $\left|\boldsymbol{E}_{m}\right|$ is $|\boldsymbol{E}|$ when $\boldsymbol{\xi}^{(m)}$ is used for the FDTD computation and $\boldsymbol{1}$ is an $\mathcal{L} \times \mathcal{L}$ identity matrix as in $\mathbf{1}=\left[\begin{array}{cccc}1 & 0 & \ldots & 0 \\ 0 & 1 & \ldots & 0 \\ \vdots & \vdots & \ddots & \vdots \\ 0 & 0 & \ldots & 1\end{array}\right]$. When $v=0$, the calculation of $\boldsymbol{a}$ is reduced to the one in the OLS method as in

$$
\boldsymbol{a}=\left(\boldsymbol{\Psi}^{T} \boldsymbol{\Psi}\right)^{-1} \boldsymbol{\Psi}^{T} \mathcal{E}
$$

in which the condition of $\mathcal{M} \geq \mathcal{L}$ must be met. $v$ is determined by minimising the penalised sum of

$$
\sum_{m=1}^{\mathcal{M}}\left(\left|\boldsymbol{E}_{m}\right|^{2}-\mathcal{T}\left(\boldsymbol{\xi}^{(m)}\right)\right)^{2}+v \sum_{l=1}^{\mathcal{L}} a_{\boldsymbol{b}_{l}}{ }^{2}
$$

In $(10), v$ exerts influence on the value of $\boldsymbol{a}$. Different values of $v$ lead to different $\boldsymbol{a}$ in 10$)$, and thus affect $\mathcal{T}\left(\boldsymbol{\xi}^{(m)}\right)$ in 9 . $v=0$ may not minimise (13) because the condition of $v=0$ may result in a larger value of $\sum_{m=1}^{\mathcal{M}}\left(\left|\boldsymbol{E}_{m}\right|^{2}-\mathcal{T}\left(\boldsymbol{\xi}^{(m)}\right)\right)^{2}$ in (13) than that in case of $v \neq 0$. Minimising 13 is equivalent to finding an optimum value of $v$ that enables the estimation of $\boldsymbol{a}$ to reach the stable status.

We use the Leave-One-Out Cross-Validation (LOOCV) method to identify the stable status of $\boldsymbol{a}$ at which the LeaveOne-Out (LOO) error $\varepsilon_{l}$ of $\mathcal{T}\left(\boldsymbol{\xi}^{(m)}\right)$ becomes minimum. At each scanned $v, \boldsymbol{a}$ is calculated in (10), and $\varepsilon_{l}$ is computed as in

$$
\varepsilon_{l}=\frac{1}{\mathcal{M}} \sum_{m=1}^{\mathcal{M}}\left(\frac{\left|\boldsymbol{E}_{m}\right|^{2}-\mathcal{T}\left(\boldsymbol{\xi}^{(m)}\right)}{1-h_{m}}\right)^{2},
$$

where $h_{m}$ is the $m$-th diagonal element of the square matrix $\boldsymbol{\Psi}\left(\boldsymbol{\Psi}^{T} \boldsymbol{\Psi}\right)^{-1} \boldsymbol{\Psi}^{T}$. The process of calculating $\boldsymbol{a}$ and $\varepsilon_{l}$ using $v$ is called a $\mathrm{RR}$ iteration. After scanning $v$, we choose the $v$, whose corresponding $\varepsilon_{l}$ is the smallest, as the optimum value. Once $v$ is determined, $\boldsymbol{a}$ is calculated in (10), and the standard deviation $\sigma$ of $|\boldsymbol{E}|^{2}$ is calculated by

$$
\sigma^{2}=\sum_{l=1}^{\mathcal{L}} a_{\boldsymbol{b}_{l}}{ }^{2}
$$

The range setting of $v$ depends upon the experimental design. A large value of $v$ will lead to a large value of the penalty term of $v \sum_{l=1}^{\mathcal{L}} a_{\boldsymbol{b}_{l}}{ }^{2}$ in $(13)$ and a small value of $\boldsymbol{a}$ [61]. Thus the estimation of the variance will become smaller than its true value. Therefore, in general, $v$ tends to be small [47].

\section{A proposed method for uncertainty quantification}

This section proposes an UQ method, aiming at the quantification of the uncertainty of the FDTD results with the minimum computational cost whilst ensuring the accuracy of the UQ results, based on the PCA and RR methods, herein named as the PCA-RR based method. It follows the principle of statistical parsimony [62] stating that a regression model should be built using only significant PC bases thereby decreasing the complexity and increasing the stability of the regression model. The procedure of the PCA-RR based method is as follows.

1) Initialisation.

a) Setting $\mathcal{M}$ to $\mathcal{K}(\mathcal{M}=\mathcal{K})$ to minimise the computational cost. For successful eigen-decomposition in PCA, $\mathcal{M}$ should be no less than $\mathcal{K}$.

b) Building $\mathcal{X}$ in $(4)$ with $\mathcal{K}$ parameters, each of which contains $\mathcal{M}$ samples chosen by LHS.

c) Constructing $\mathcal{E}$ in 11 with $\mathcal{M}|\boldsymbol{E}|^{2}$ obtained from $\mathcal{M}$ FDTD simulations using $\mathcal{M} \boldsymbol{\xi}^{(m)}$.

2) Transformation of $\mathcal{X}$ to $\mathcal{X}_{\text {sub }}$.

Utilising the PCA method to keep $\mathcal{D}$ parameters and construct the sample set $\mathcal{X}_{\text {sub }}$ in 8 .

3) Sparse PC expansion. 
Building the sparse PC expansion in (9) by replacing $\mathcal{K}$ with $\mathcal{D}$ in Section $\Pi-\mathrm{A}$.

4) Calculation of $\boldsymbol{a}$.

When $\mathcal{M} \geq \mathcal{L}$, we calculate $\boldsymbol{a}$ by the OLS method as in

$$
\boldsymbol{a}=\left(\hat{\boldsymbol{\Psi}}^{T} \hat{\boldsymbol{\Psi}}\right)^{-1} \hat{\boldsymbol{\Psi}}^{T} \mathcal{E},
$$

where the PC bases matrix $\hat{\Psi}=\left(\psi_{\boldsymbol{b}_{l}}\left(\hat{\boldsymbol{\xi}}^{(m)}\right), m=1 \sim\right.$ $\mathcal{M}, l=1 \sim \mathcal{L})$. Otherwise the RR method is utilised to calculate $\boldsymbol{a}$ as in

$$
\boldsymbol{a}=\left(\hat{\boldsymbol{\Psi}}^{T} \hat{\boldsymbol{\Psi}}+v \boldsymbol{1}\right)^{-1} \hat{\boldsymbol{\Psi}}^{T} \mathcal{E},
$$

where $v$ is scanned and set to the one when

$$
\varepsilon_{l}=\frac{1}{\mathcal{M}} \sum_{m=1}^{\mathcal{M}}\left(\frac{\left|\boldsymbol{E}_{m}\right|^{2}-\mathcal{T}\left(\hat{\boldsymbol{\xi}}^{(m)}\right)}{1-\hat{h}_{m}}\right)^{2}
$$

reaches its minimum. $\hat{h}_{m}$ is the $m$-th diagonal element of the square matrix $\hat{\mathbf{\Psi}}\left(\hat{\mathbf{\Psi}}^{T} \hat{\mathbf{\Psi}}\right)^{-1} \hat{\mathbf{\Psi}}^{T}$.

5) The average value and the standard deviation.

We calculate the average value $\mu$ of $|\boldsymbol{E}|^{2}$ as in

$$
\mu=\frac{1}{\mathcal{M}} \sum_{m=1}^{\mathcal{M}}\left|\boldsymbol{E}_{m}\right|^{2},
$$

and calculate $\sigma$ in 15 .

The PCA-RR based method is summarised in Fig. 1

The PCA-RR method offers two advantages which allow us to minimise the computational cost of UQ while maintaining the accuracy of the UQ results.

1) Before building the sparse PC expansion, the PCA method is utilised to reduce a certain number of input parameters. As a consequence, we obtain the less number of PC bases than in the PC expansion without using the PCA method. The fewer number of PC bases required for the UQ computation means the lower complexity of the regression model thereby improving the stability and accuracy of UQ results.

2) Although the RR method is more versatile than the OLS method, it has a higher algorithmical complexity compared to the OLS method and requires more computational resources. The condition of $\mathcal{M} \geq \mathcal{L}$ in Fig. 1 enables to improve the computational efficiency for UQ. When $\mathcal{M} \geq \mathcal{L}$, the RR method is not the preferred method to calculate $a$, rather the OLS method in (16) is then best suited. As a consequence, the unnecessary computation of using RR method is avoided and the computational cost of the PCA-RR based method is reduced.

\section{NUMERICAL EXPERIMENTS FOR UNCERTAINTY QUANTIFICATION}

The MCM and the PCA-RR based method are utilised to quantify the uncertainty of the FDTD results. The simulation scenario is depicted in Fig. 2 where we place a point soft source excitation $17 \mathrm{~mm}$ away from the human body and observe $\boldsymbol{E}$ in the middle of the prostate tissue. 10 layers of the complex frequency shifted-perfect matched layers (CFS-PML)

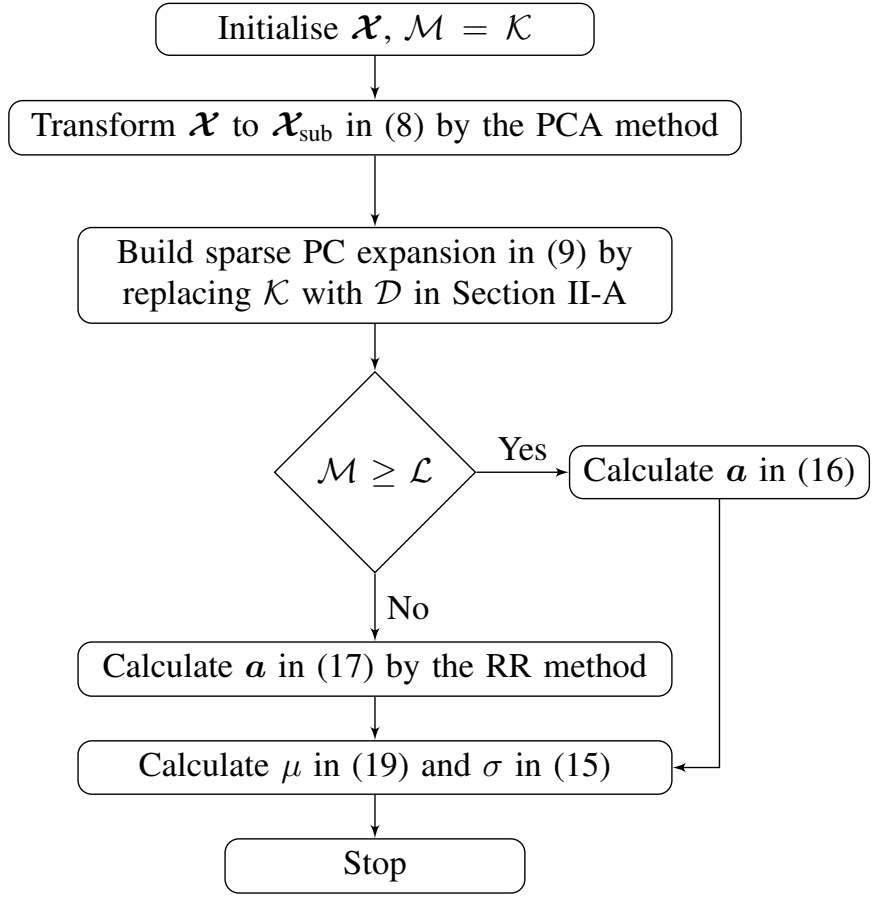

Fig. 1. Flow chart of the PCA-RR based method for UQ

[63] [64] are used to terminate the $265 \times 490 \times 601$ FDTD space. The digital human phantom (DHP) used in this work is provided by RIKEN (Saitama, Japan) under the non-disclosure agreement between RIKEN and the University of Manchester. The usage was approved by RIKEN ethical committee.

We use $|\boldsymbol{E}|^{2}$ as the output of the 3-dimensional FDTD simulation calculated as in $|\boldsymbol{E}|^{2}=$ $\sum_{n=1}^{5000}\left(\left|E_{x}^{n}(i, j, k)\right|^{2}+\left|E_{y}^{n}(i, j, k)\right|^{2}+\left|E_{z}^{n}(i, j, k)\right|^{2}\right), \quad$ where $(i, j, k)$ is the point location of the observation in the prostate and $n$ is the FDTD time step. There are five influential tissues (fat, skin, muscle, bone and prostate) between the excitation and observation. $|\boldsymbol{E}|^{2}$ observed changes significantly when one or more of the Debye parameters of the influential tissues are changed. The Debye parameters for all human tissues are presented in [65]. In our scenario, the input parameters refer to the Debye parameters of interest of the influential tissues whose complex permittivity and conductivity are varied within $\pm 10 \%$ in the FDTD simulations. The remaining Debye parameters of the human tissues as well as the values associated with the setting of the FDTD simulations, such as temporal and spatial sampling rate, are treated as constants.

1) The Monte Carlo method

The details of utilising the MCM for UQ are explained in [41], where $\mu(\mathcal{M})$ and $\sigma(\mathcal{M})^{2}$ are calculated by

$$
\mu(\mathcal{M})=\frac{1}{\mathcal{M}} \sum_{m=1}^{\mathcal{M}}\left|\boldsymbol{E}_{m}\right|^{2}
$$

and

$$
\sigma(\mathcal{M})^{2}=\frac{1}{\mathcal{M}-1} \sum_{m=1}^{\mathcal{M}}\left(\left|\boldsymbol{E}_{m}\right|^{2}-\mu(\mathcal{M})\right)^{2}
$$




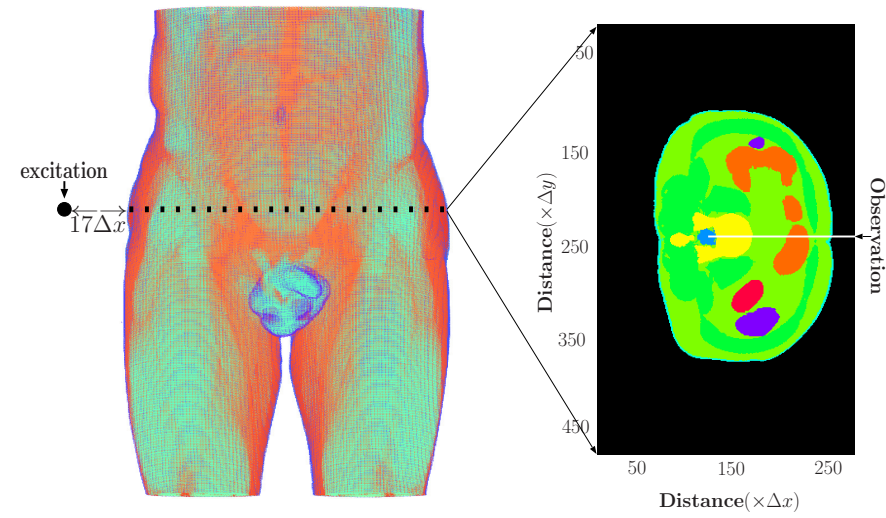

Fig. 2. Setup for the FDTD computation

using the first $\mathcal{M}|\boldsymbol{E}|^{2}$ among the $10^{4}|\boldsymbol{E}|^{2}$ varying $\mathcal{M}$ from 2 to $10^{4}$.

2) The PCA-RR based method

In the PCA method, $5 \%$ of information $\left(\widehat{\mathcal{C}}_{\mathcal{D}} \geq 95 \%\right)$ [21] can be safely dropped without significantly affecting the accuracy of PCA. For example, in one of numerical experiments in case of $\mathcal{K}=10$ and $\mathcal{M}=$ 10,10 eigenvalues are obtained from (5) as in $\widetilde{\mathcal{H}}=$ $\left\{3.2,2.2,1.3,1.3,1.0,7.0 \cdot 10^{-1}, 2.3 \cdot 10^{-1}, 6.3 \cdot 10^{-2} 1.4\right.$. $\left.10^{-2}, 1.1 \cdot 10^{-16}\right\} . \mathcal{C}$ and $\widehat{\mathcal{C}}$ are then calculated in $(6)$ and (7), respectively. The results are presented in Fig. 3

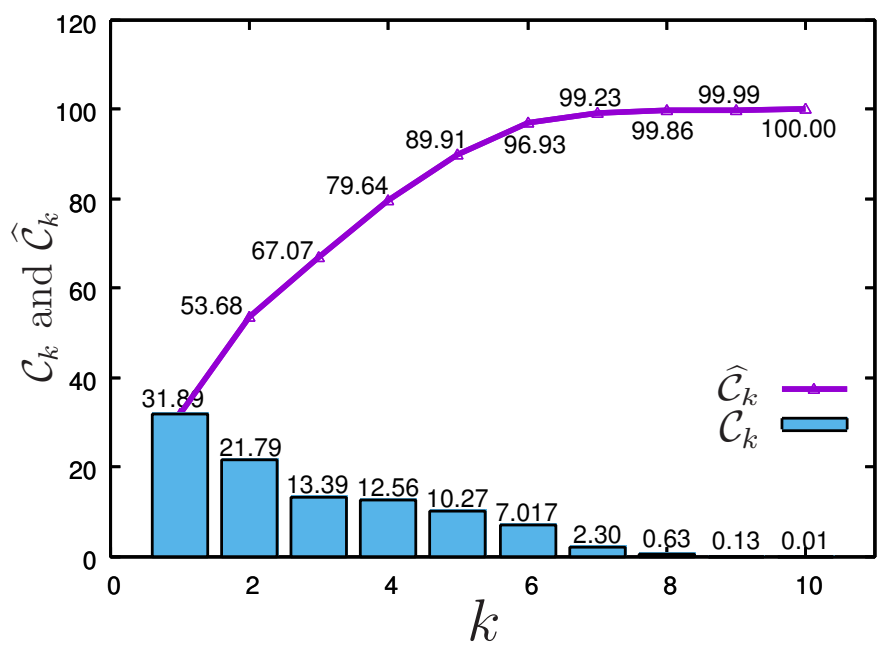

Fig. 3. $\mathcal{C}_{k}$ and $\widehat{\mathcal{C}}_{k}$ in case of $\mathcal{K}=10$ and $\mathcal{M}=10$.

Fig. 3 illustrates that $31.89 \%$ information can be explained by the first principal component, and contribution of each principal component to the total information is gradually reduced with the growth of $k$. Furthermore, the first $6(\mathcal{D}=6)$ principal components possess over 95\% information $\left(\widehat{\mathcal{C}}_{6}=96.93 \%\right)$. Therefore, the first 6 principal components are kept.

We obtain $\mathcal{L}=12$ by replacing $\mathcal{K}$ in Section $\mathrm{II}-\mathrm{A}$ with $\mathcal{D}$ in the case of $\mathcal{D}=6$ and $r=2$. The condition of $\mathcal{M} \geq \mathcal{L}$ is not met. Thus the RR method is required for the calculation of $\boldsymbol{a}$. Fig. 4 shows the change of the first 5 $a_{\boldsymbol{b}_{l}}$ as $v$ increases from $10^{-6}$ to $2 \cdot 10^{-3}$ with an interval of $10^{-6}$. At some point, these coefficients become stable and gradually shrink to zero.

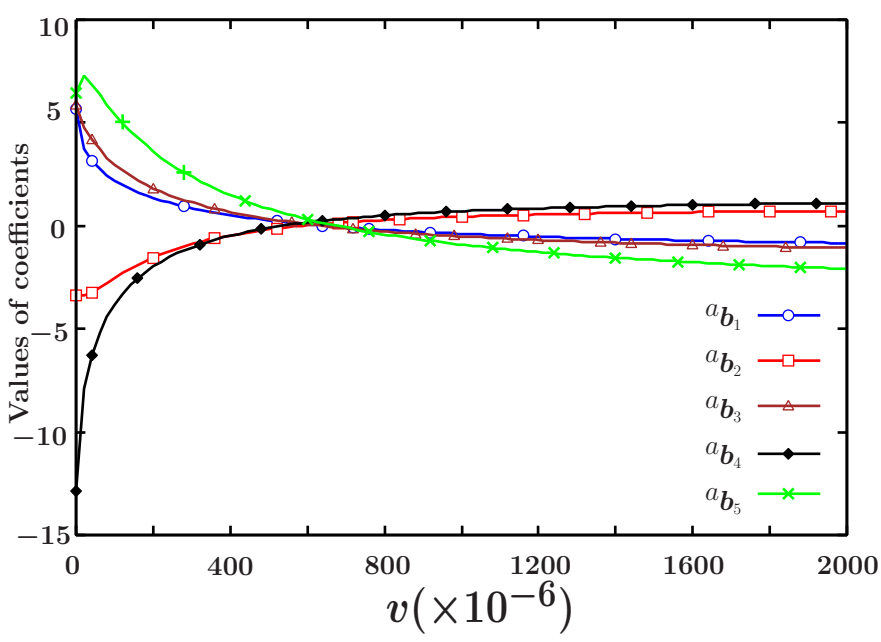

Fig. 4. The change of the first $5 a_{\boldsymbol{b}_{l}}$ with the increase of $v$ from $10^{-6}$ to $2 \cdot 10^{-3}$ with an interval of $10^{-6}$.

Fig. 5 is $\varepsilon_{l}$ calculated using $\boldsymbol{a}$ in Fig. 4. $\varepsilon_{l}$ is minimum when $v=2.2 \cdot 10^{-4}$. We therefore choose $\boldsymbol{a}$ calculated with $v=2.2 \cdot 10^{-4}$. The $\sigma$ of $|\boldsymbol{E}|^{2}$ is then estimated in 15 using the chosen $\boldsymbol{a}$ and the $\mu$ of $|\boldsymbol{E}|^{2}$ is estimated in 20 using the $10|\boldsymbol{E}|^{2}$ obtained from the 10 FDTD simulations.

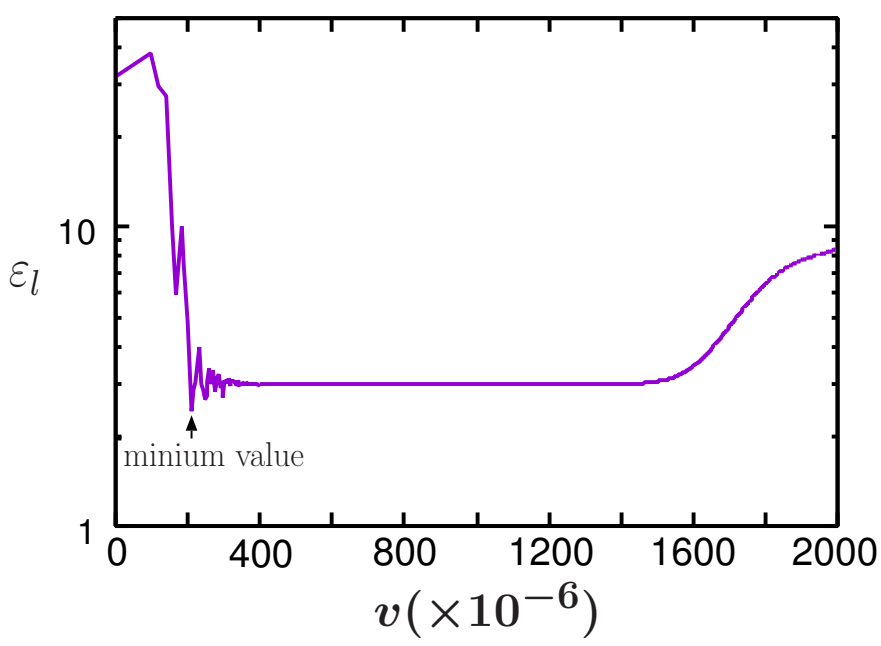

Fig. 5. The change of $\varepsilon_{l}$ with the increase of $v$ from $10^{-6}$ to $2 \times 10^{-3}$ with an interval of $10^{-6}$.

\section{RESULTS AND DISCUSSIONS}

Section IV presents $\mu$ and $\sigma$ obtained from the MCM and the PCA-RR based methods. Furthermore, a discussion is carried out to emphasise the merit of the PCA-RR based method.

\section{A. Results}

1) Average values: 


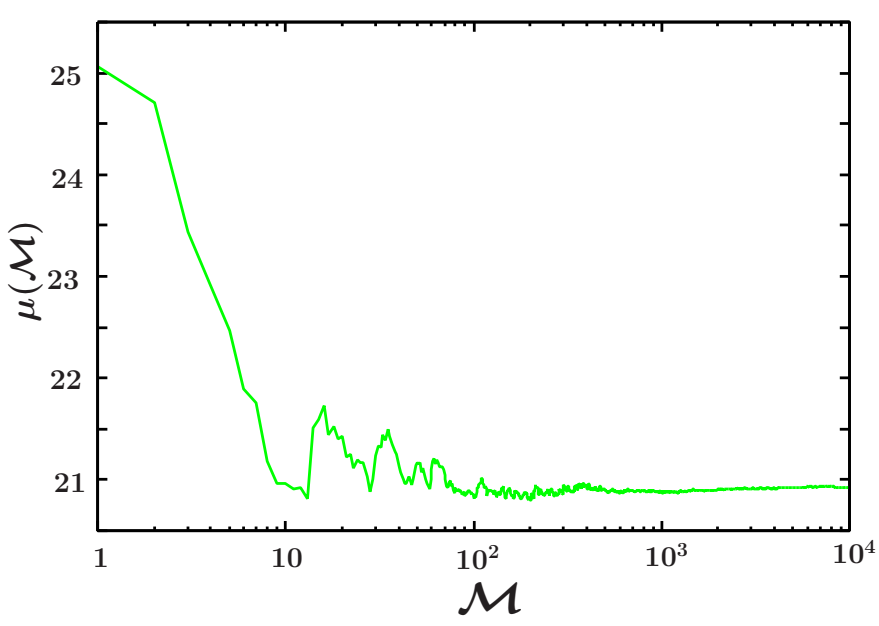

Fig. 6. $\mu(\mathcal{M})$ in 20 from the MCM, which is the same as Fig. 6 in [41].

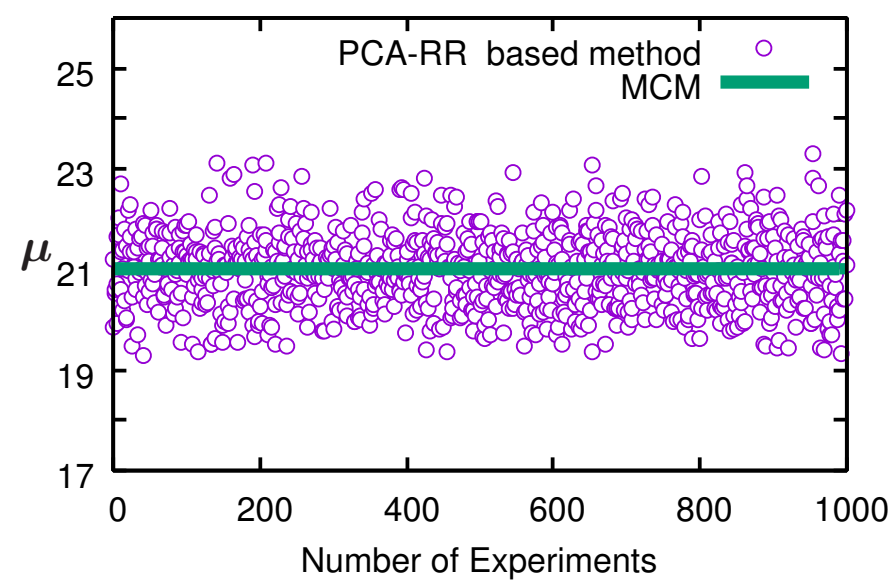

Fig. 7. $\mu$ from the PCA-RR based method for 1000 experiments and $\mu\left(10^{4}\right)$ from the MCM.

The $\mu(\mathcal{M})$ of the first $\mathcal{M}|\boldsymbol{E}|^{2}$ obtained from the MCM is presented in Fig. 6, where $\mathcal{M}$ varies from 2 to $10^{4}$. Fig. 7 shows $\mu$ obtained from the PCA-RR based methods for 1000 experiments. The $\mu$ from the MCM when $\mathcal{M}=10^{4}$ is presented as a straight line for comparison.

2) Standard deviations:

Fig. 8 presents $\sigma(\mathcal{M})$ of the first $\mathcal{M}|\boldsymbol{E}|^{2}$ obtained from the MCM, where $\mathcal{M}$ varies from 2 to $10^{4}$.

Fig. 9 shows $\sigma$ obtained from the PCA-RR based method for 1000 experiments. $\sigma$ from the MCM when $\mathcal{M}=10^{4}$ is presented as a straight line for comparison.

\section{B. Discussions}

The PCA-RR based method focuses on the improvement of the computational efficiency while preserving the accuracy of the UQ result. The performance of the PCA-RR based method is analysed from the viewpoint of its accuracy and the computational efficiency.

1) Accuracy:

We define the result from the PCA-RR based method less than $\pm 5 \%$ away from the result of MCM as the satisfactory

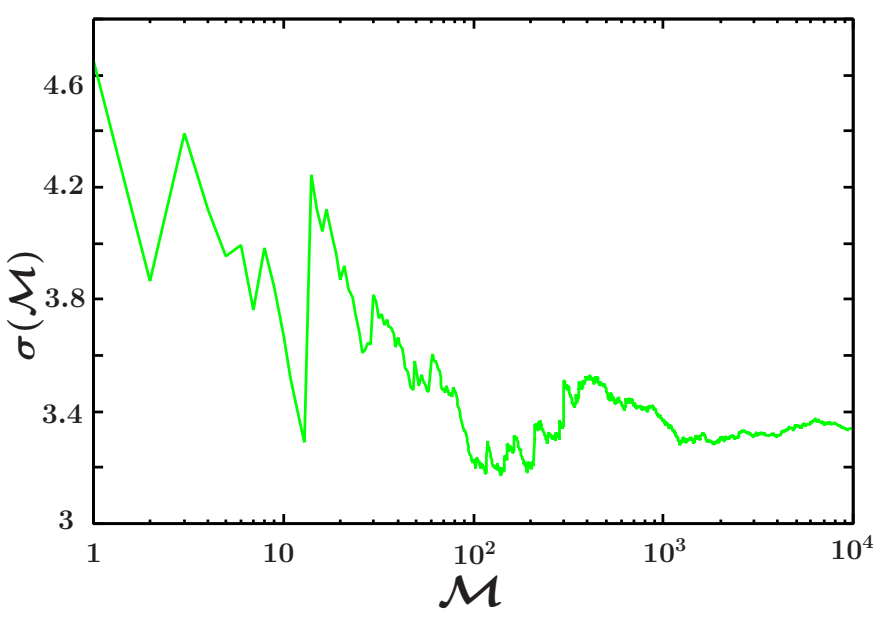

Fig. 8. $\sigma(\mathcal{M})$ in 21 from the MCM, which is the same as Fig. 8 in [41].

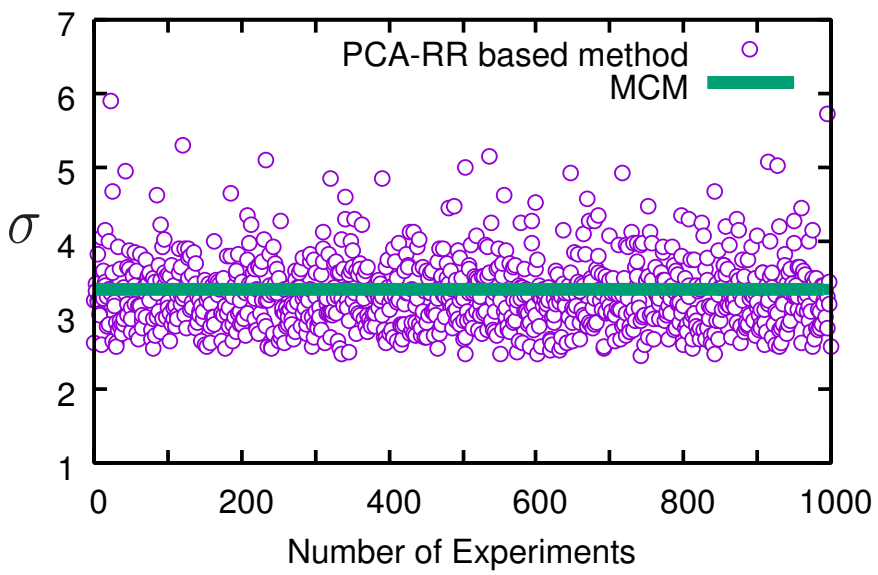

Fig. 9. $\sigma$ from the PCA-RR based method for 1000 experiments and $\sigma\left(10^{4}\right)$ from the MCM.

result. $875 \mu$ out of $1000 \mu$ in Fig. 7 and $722 \sigma$ out of 1000 $\sigma$ in Fig. 9 are satisfactory. The accuracies of using PCARR based method for UQ of the FDTD results are therefore $87.5 \%$ for average estimation and $72.2 \%$ for standard deviation estimation, which outperforms one of the state-of-the-art UQ techniques namely the least angle regression (LARS) method whose accuracies are $86.8 \%$ for average estimation and $63.4 \%$ for standard deviation estimation [41]. The main causes of unsatisfactory results from the PCA-RR based method include underfitting, outlier and unreasonable $\varepsilon_{l}$. The underfitting and the outlier have been detailed in our previous work [41]. As for the unreasonable $\varepsilon_{l}$, in the process of the RR method, $v$ is determined at which $\varepsilon_{l}$ is minimum. We randomly choose the 100 satisfactory $\sigma$ and 100 unsatisfactory $\sigma$ in Fig. 9 and present their corresponding minimum $\varepsilon_{l}$ in Fig. 10. It is worth noting that minimum $\varepsilon_{l}$ of the 100 unsatisfactory $\sigma$ are about 6 times higher than those of the 100 satisfactory $\sigma$. We call the $\varepsilon_{l}$ larger than 0.5 as the unreasonable $\varepsilon_{l}$. In the RR method, all 2000 scanned $v$ could yield unreasonable $\varepsilon_{l}$. In such a case, the minimum $\varepsilon_{l}$ is also unreasonable. An unreasonable $\varepsilon_{l}$ indicates that the calculation of $\boldsymbol{a}$ in the PCA-RR based method is not accurate and thus the UQ result deviates from 


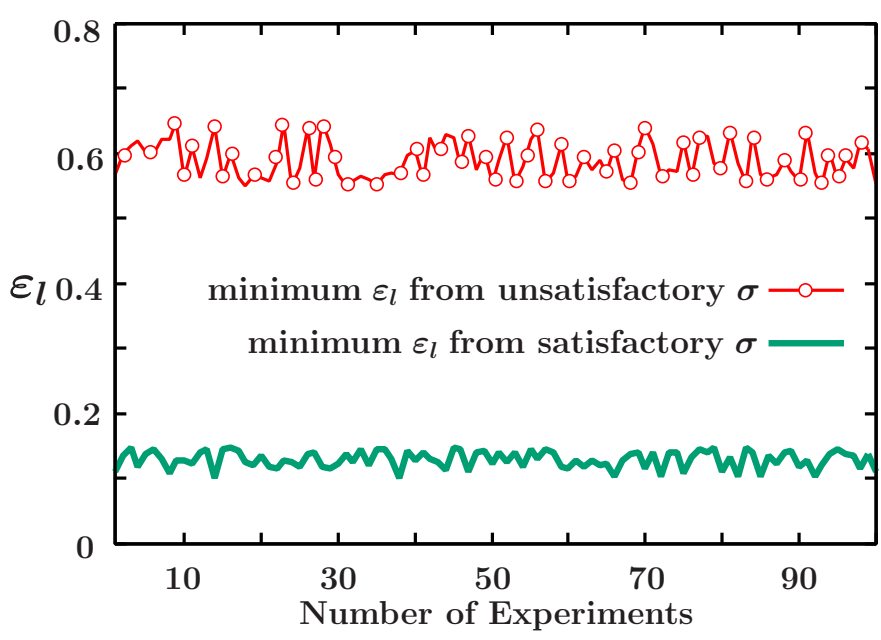

Fig. 10. Minimum $\varepsilon_{l}$ of 100 satisfactory $\sigma$ and 100 unsatisfactory $\sigma$.

its true value.

Furthermore, we evaluate the influence of the LHS method on the accuracy and stability of the PCA-RR based method. The average and the standard deviation of $\sigma$ in Fig. 9, which are obtained from the PCA-RR based method with the LHS method, are $3.395 \pm 0.349$, while $\sigma\left(10^{4}\right)=3.348$. Replacing the LHS method with the random sampling (RS) method, we conduct 1000 experiments to obtain $1000 \sigma$ using the PCARR based method. The average and the standard deviation for the $1000 \sigma$ are presented in Table I] which demonstrates that the LHS method improves both accuracy and stability of the PCA-RR based method.

\begin{tabular}{|c|c|c|c|}
\hline & Accuracy & Stability \\
\hline & $\begin{array}{c}\text { Average } \\
\text { of } \sigma\end{array}$ & $\begin{array}{c}\text { Standard } \\
\text { deviation of } \sigma\end{array}$ \\
\hline $\begin{array}{c}\text { PCA-RR based } \\
\text { method with LHS }\end{array}$ & 3.395 & $98.59 \%$ & 0.349 \\
\hline $\begin{array}{c}\text { PCA-RR based } \\
\text { method with RS }\end{array}$ & 3.237 & $96.68 \%$ & 0.475 \\
\hline
\end{tabular}

COMPARISON OF THE LHS METHOD AND THE RS METHOD. THE ACCURACY IS CALCULATED AS IN $1-\frac{\left|\frac{1}{1000} \sum \sum^{1000} \sigma-\sigma\left(10^{4}\right)\right|}{\sigma\left(10^{4}\right)}$.

\section{2) Computational efficiency:}

Apart from the FDTD simulation, the computational cost of an UQ technique is negligible. Therefore, we only take the number of required FDTD simulations into account when evaluating the computational efficiency of an UQ technique. Fig. 11] shows the number of required FDTD simulations with the increase of $\mathcal{K}$ in the three methods of the general PC expansion, the sparse PC expansion and the PCA-RR based method, where the general PC expansion refers to the PC expansion with $\mathcal{L}$ calculated in (2) without utilising the hyperbolic scheme. The PCA-RR based method demands the smallest number of FDTD simulations among the three techniques thereby greatly reducing the computational cost. For example, when $\mathcal{K}=10$, the number of required FDTD

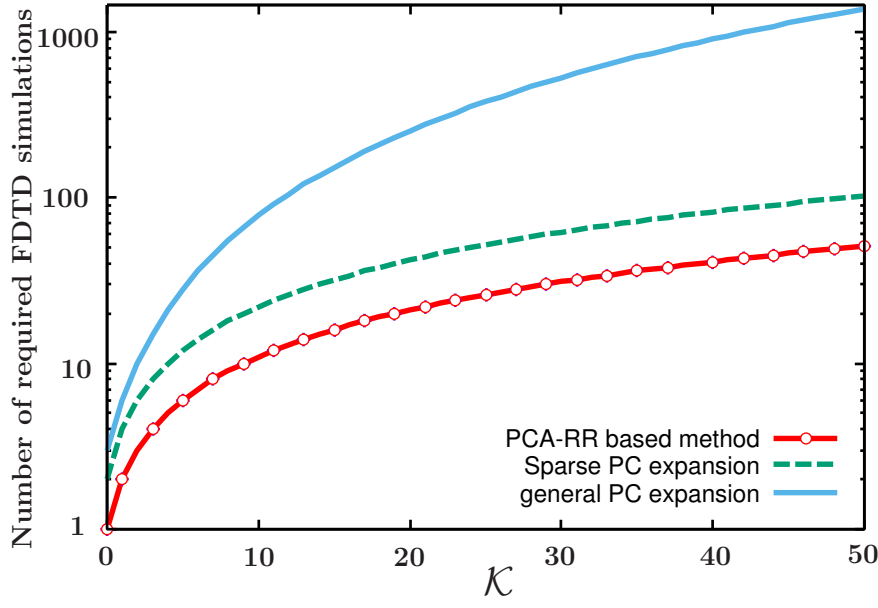

Fig. 11. The number of required FDTD simulations with the increase of $\mathcal{K}$ calculated in Step 1 in Section III-D for the PCA-RR based method, 3 for the sparse PC expansion in the case of $r=2$ and $q=0.4$, and (2) for the general PC expansion in the case of $r=2$.

simulations in the general PC expansion and the sparse PC expansion are 66 and 20 respectively, whereas the PCA-RR based method only requires 10 FDTD simulations. In general, when $q=0.4$, the computational cost with regard to the FDTD simulations in the PCA-RR based method is half of the cost required in the sparse PC expansion.

Though the minimum number of required FDTD simulations in the PCA-RR based method is identical to the number of input parameters, the accuracy of UQ results can be further improved from additional input samples. Fig. 12 shows $\mu$ and $\sigma$ obtained from the PCA-RR based method varying $\mathcal{M}$ from 10 to 100 . The $\mu$ and $\sigma$ from the MCM when $\mathcal{M}=10^{4}$ are presented as dashed lines for comparison. When $\mathcal{M} \geq 30, \sigma$ is stable as $\mathcal{M}$ increases. Fig. 13 shows the influence of $v$ on $\sigma$ in 15 in the case of $\mathcal{M}=10$, where $v$ varies from $10^{-7}$ to 0.5 . With the increase of $v, \boldsymbol{a}$ in (17) gradually decreases resulting in the reduction of $\sigma$ in (15).

We define the PCA-RR based method with the minimum $\varepsilon_{l}$ in (18) less than 0.1 as the high-accuracy PCA-RR (HAPCA-RR) based method. Table II presents the comparisons of the proposed method with other advanced UQ techniques including the NIPC method, the works in [52] and [20], where the P-PCA-RR based method refers to the parsimonious PCARR based method which only uses the minimum number of required FDTD simulations. [52] combines PCA with sparse grid interpolation for UQ and the relative tolerance is set to 0.2 in our scenario. [20] proposes a combined uncertainty based UQ method. It is worth noting that the accuracy of $\sigma$ in HAPCA-RR based method outperforms other UQ methods while it demands the second least number of FDTD simulations. Though the performance of the P-PCA-RR based method is relatively moderate, it requires the least number of FDTD simulations.

\section{CONCLUSION}

Various sparse techniques can be utilised to alleviate the curse of dimensionality. However, the computational cost is 


\begin{tabular}{|c|c|c|c|c|c|}
\hline UQ methods & FDTD simulations & $\mu$ & Accuracy of $\mu$ & $\sigma$ & Accuracy of $\sigma$ \\
\hline NIPC & 65 & 20.942 & $99.56 \%$ & 4.083 & $78.11 \%$ \\
\hline Work in & 221 & 21.154 & $98.54 \%$ & 3.267 & $97.52 \%$ \\
\hline Work in & 30 & 20.720 & $99.37 \%$ & 4.027 & $79.79 \%$ \\
\hline P-PCA-RR based & 10 & 21.116 & $98.72 \%$ & 3.844 & $85.25 \%$ \\
\hline HA-PCA-RR based & 27 & 20.947 & $99.41 \%$ & 3.305 & $98.66 \%$ \\
\hline
\end{tabular}

COMPARISONS OF THE PROPOSED METHOD WITH THE UQ METHODS OF NIPC EXPANSION, [52] AND [20], WHERE P-PCA-RR REFERS TO THE PARSIMONIOUS PCA-RR BASED METHOD WHICH ONLY USES THE MINIMUM NUMBER OF REQUIRED FDTD SIMULATIONS, AND HA-PCA-RR INDICATES THE HIGH-ACCURACY PCA-RR BASED METHOD WHOSE MINIMUM $\varepsilon_{l}$ IN 18 IS LESS THAN 0.1. THE ACCURACIES ARE CALCULATED BY $1-\frac{\left|\mu-\mu\left(10^{4}\right)\right|}{\mu\left(10^{4}\right)}$ AND $1-\frac{\left|\sigma-\sigma\left(10^{4}\right)\right|}{\sigma\left(10^{4}\right)}$, RESPECTIVELY.

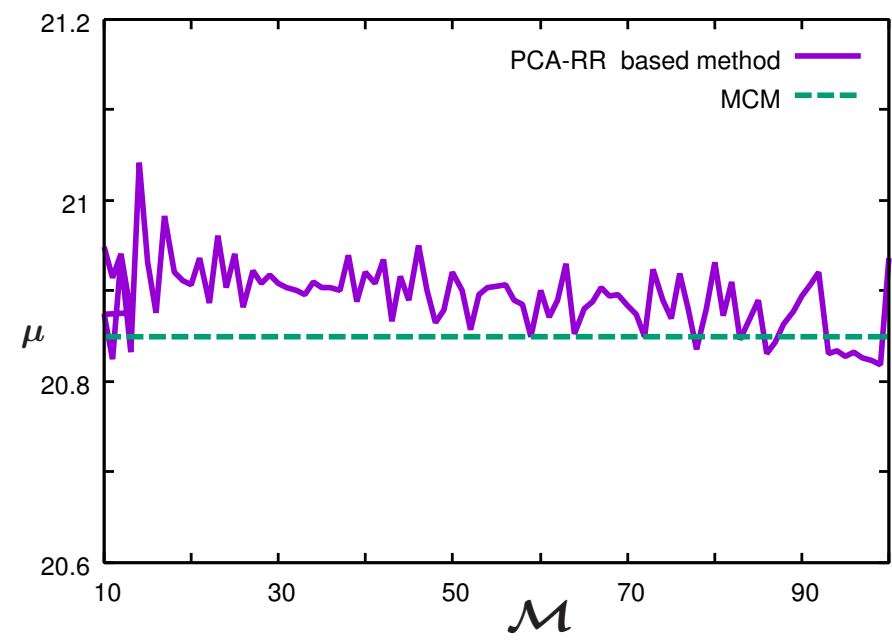

(a) $\mu$ in 19 from the PCA-RR based method varying $\mathcal{M}$ from 10 to 100.

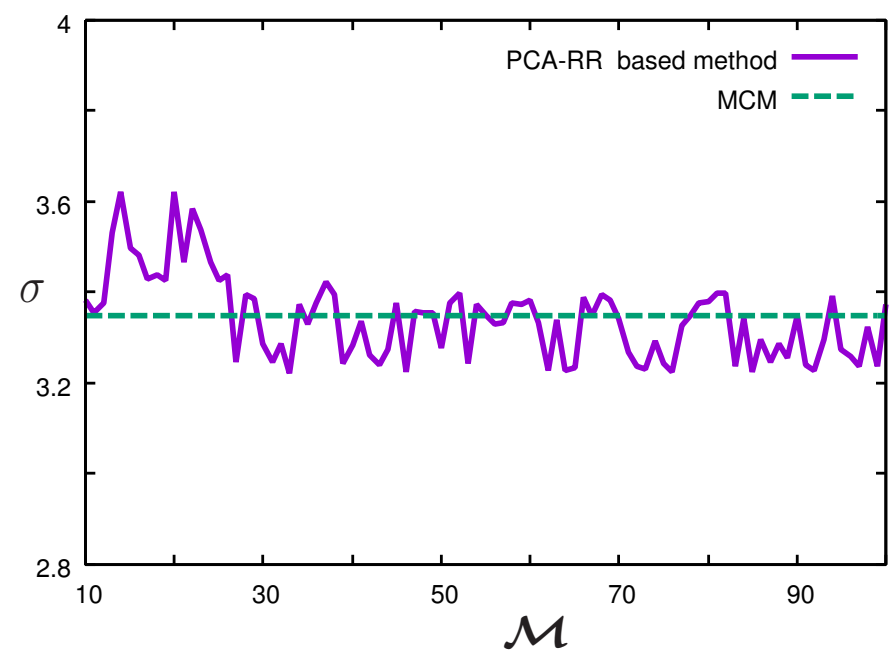

(b) $\sigma$ in 15 from the PCA-RR based method varying $\mathcal{M}$ from 10 to 100 .

Fig. 12. Change of $\mu$ and $\sigma$ from the PCA-RR based method with the increase of $\mathcal{M}$.

still non-negligible when a system has a considerable number of input parameters. This paper proposed a versatile UQ technique in order to overcome this problem. In contrast to the previous works on UQ, the PCA-RR based method significantly reduces the number of required FDTD simulations by combining the PCA method and the hyperbolic scheme. It also introduces the RR method in the calculation of the NIPC coefficients to enable the matrix inversion to be successfully performed irrespective of the number of input

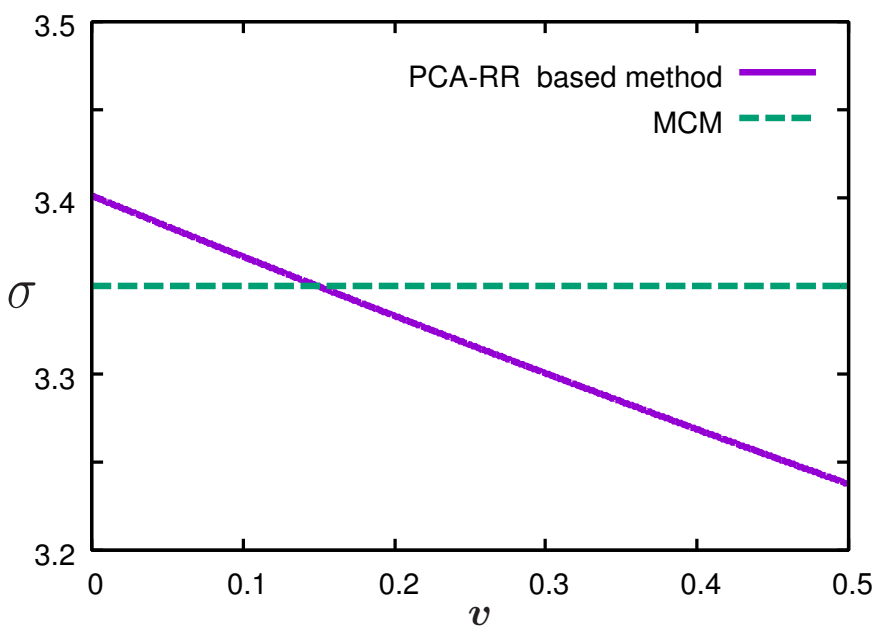

Fig. 13. $\quad \sigma$ with the increase of $v$ when $\mathcal{M}=10$.

samples. As a consequence, the PCA-RR based method is capable of accurately quantifying the uncertainty of the FDTD computation using only 10 FDTD simulations for 10 input Debye-parameters, whereas at least 66 FDTD simulations are required in the general NIPC expansion.

The main contribution of this paper lies in the significant reduction of the computational cost for UQ. Comparing with existing UQ techniques, the PCA-RR based method is one of the least-costly UQ techniques. Furthermore, it presents a better stability than other regression techniques such as the LARS method and the least absolute shrinkage and selection operator (LASSO) method [66] since our method reduces the complexity of the regression model.

\section{ACKNOWLEDGEMENT}

This work was completed in part with HOKUSAIGreatWave Computer System at RIKEN. http://i.riken.jp/ download/sites/2/HOKUSAI_system_overview_en.pdf

\section{REFERENCES}

[1] K. Sairam, N. Gunasekaran, and S. R. Redd, "Bluetooth in wireless communication," IEEE Commun. Mag., vol. 40, no. 6, pp. 90-96, 2002.

[2] A. Taflove and S. C. Hagness, Computational electrodynamics: the finitedifference time-domain method. Artech house, 2005.

[3] K. Yee, "Numerical solution of initial boundary value problems involving maxwell's equations in isotropic media," IEEE Trans. Antennas Propag., vol. 14, no. 3, pp. 302-307, 1966. 
[4] K. Tekbas, F. Costen, J.-P. Bérenger, R. Himeno, and H. Yokota, "Subcell modeling of frequency-dependent thin layers in the FDTD method," IEEE Trans. Antennas Propag., vol. 65, no. 1, pp. 278-286, 2017.

[5] R. G. Ghanem and P. D. Spanos, "Stochastic finite element method: Response statistics," in Stochastic Finite Elements: A Spectral Approach, pp. 101-119, Springer, 1991.

[6] O. Aiouaz, D. Lautru, M.-F. Wong, E. Conil, A. Gati, J. Wiart, and V. F Hanna, "Uncertainty analysis of the specific absorption rate induced in a phantom using a stochastic spectral collocation method," Annals of Telecommunications-annales des télécommunications, vol. 66, no. 7-8, pp. 409-418, 2011.

[7] M. Cvetkovič, S. Lallechere, K. E. Khamlichi Drissi, P. Bonnet, and D. Poljak, "Stochastic sensitivity in homogeneous electromagneticthermal dosimetry model of human brain," Applied Computational Electromagnetics Society Journal, vol. 31, no. 6, 2016.

[8] L. J. Gomez, A. C. Yücel, L. Hernandez-Garcia, S. F. Taylor, and E. Michielssen, "Uncertainty quantification in transcranial magnetic stimulation via high-dimensional model representation," IEEE Trans. Biomed. Eng., vol. 62, no. 1, pp. 361-372, 2015.

[9] S. Weinzierl, "Introduction to monte carlo methods," NIKHEF, Amsterdam, The Netherlands, Tech. Rep. NIKHEF-00-012, 2000.

[10] A. Arduino, M. Chiampi, F. Pennecchi, L. Zilberti, and O. Bottauscio, "Monte carlo method for uncertainty propagation in magnetic resonancebased electric properties tomography," IEEE Trans. Magn., vol. 53, no. 11, pp. 1-4, 2017.

[11] C. Yardim, P. Gerstoft, and W. S. Hodgkiss, "Estimation of radio refractivity from radar clutter using Bayesian Monte Carlo analysis," IEEE Trans. Antennas Propag., vol. 54, no. 4, pp. 1318-1327, 2006.

[12] D. Xiu and J. S. Hesthaven, "High-order collocation methods for differential equations with random inputs," SIAM Journal on Scientific Computing, vol. 27, no. 3, pp. 1118-1139, 2005.

[13] M. A. Tatang, W. Pan, R. G. Prinn, and G. J. McRae, "An efficient method for parametric uncertainty analysis of numerical geophysical models," Journal of Geophysical Research: Atmospheres, vol. 102, no. D18, pp. 21925-21932, 1997.

[14] L. Mathelin, M. Y. Hussaini, and T. A. Zang, "Stochastic approaches to uncertainty quantification in CFD simulations," Numerical Algorithms, vol. 38, no. 1-3, pp. 209-236, 2005.

[15] N. Wiener, "The homogeneous chaos," American Journal of Mathematics, vol. 60, no. 4, pp. 897-936, 1938

[16] D. Xiu and G. E. Karniadakis, "The Wiener-Askey polynomial chaos for stochastic differential equations," SIAM journal on scientific computing, vol. 24, no. 2, pp. 619-644, 2002.

[17] D. Xiu and G. E. Karniadakis, "Modeling uncertainty in flow simulations via generalized polynomial chaos," Journal of computational physics, vol. 187 , no. 1, pp. 137-167, 2003 .

[18] A. J. Chorin, "The numerical solution of the navier-stokes equations for an incompressible fluid," Bulletin of the American Mathematical Society, vol. 73, no. 6, pp. 928-931, 1967.

[19] I. Jeong, B.-G. Gu, J. Kim, K. Nam, and Y. Kim, "Inductance estimation of electrically excited synchronous motor via polynomial approximations by least square method," IEEE Trans. Ind. Appl., vol. 51, no. 2, pp. 1526-1537, 2015

[20] X. Cheng and V. Monebhurrun, "Application of different methods to quantify uncertainty in specific absorption rate calculation using a cadbased mobile phone model," IEEE Trans. Electromagn. Compat., vol. 59, no. 1, pp. 14-23, 2017.

[21] G. Blatman and B. Sudret, "Adaptive sparse polynomial chaos expansion based on least angle regression," Journal of Computational Physics, vol. 230, no. 6, pp. 2345-2367, 2011.

[22] B. Sudret, "Polynomial chaos expansions and stochastic finite element methods," Risk and reliability in geotechnical engineering, pp. 265-300, 2014.

[23] G. E. Box, J. S. Hunter, and W. G. Hunter, Statistics for experimenters: design, innovation, and discovery, vol. 2. Wiley-Interscience New York, 2005.

[24] I. M. Sobol, "Global sensitivity indices for nonlinear mathematical models and their Monte Carlo estimates," Mathematics and computers in simulation, vol. 55, no. 1-3, pp. 271-280, 2001.

[25] X. Yang, M. Choi, G. Lin, and G. E. Karniadakis, "Adaptive ANOVA decomposition of stochastic incompressible and compressible flows," Journal of Computational Physics, vol. 231, no. 4, pp. 1587-1614, 2012

[26] X. Ma and N. Zabaras, "An adaptive high-dimensional stochastic model representation technique for the solution of stochastic partial differential equations," Journal of Computational Physics, vol. 229, no. 10 pp. 3884-3915, 2010
[27] Z. Zhang, X. Yang, G. Marucci, P. Maffezzoni, I. A. M. Elfadel, G. Karniadakis, and L. Daniel, "Stochastic testing simulator for integrated circuits and MEMS: Hierarchical and sparse techniques," in Custom Integrated Circuits Conference (CICC), 2014 Proceedings of the IEEE, pp. 1-8, 2014.

[28] Z. Zhang, M. Choi, and G. E. Karniadakis, "Error estimates for the ANOVA method with polynomial chaos interpolation: tensor product functions," SIAM Journal on Scientific Computing, vol. 34, no. 2 , pp. A1165-A1186, 2012.

[29] A. C. Yücel, H. Bağci, and E. Michielssen, "An ME-PC enhanced HDMR method for efficient statistical analysis of multiconductor transmission line networks," IEEE Trans. Compon., Packag., Manuf. Technol., vol. 5, no. 5, pp. 685-696, 2015.

[30] Z. Zhang, X. Yang, I. V. Oseledets, G. E. Karniadakis, and L. Daniel, "Enabling high-dimensional hierarchical uncertainty quantification by ANOVA and tensor-train decomposition," IEEE Trans. Comput.-Aided Design Integr. Circuits Syst., vol. 34, no. 1, pp. 63-76, 2015.

[31] X. Li, "Finding deterministic solution from underdetermined equation: large-scale performance variability modeling of analog/RF circuits," IEEE Trans. Comput.-Aided Design Integr. Circuits Syst, vol. 29, no. 11, pp. 1661-1668, 2010.

[32] Z. Zhang, T.-W. Weng, and L. Daniel, "Big-data tensor recovery for high-dimensional uncertainty quantification of process variations," IEEE Trans. Compon., Packag., Manuf. Technol., vol. 7, no. 5, pp. 687-697, 2017.

[33] T. El-Moselhy and L. Daniel, "Variation-aware interconnect extraction using statistical moment preserving model order reduction," in Proceedings of the Conference on Design, Automation and Test in Europe, pp. 453-458, European Design and Automation Association, 2010.

[34] M. Abramowitz and I. Stegun, "Handbook of mathematical functions: With formulas, graphs, and mathematical tables applied mathematics series," National Bureau of Standards, Washington, DC, 1964.

[35] C. Cui and Z. Zhang, "Stochastic collocation with non-gaussian correlated process variations: Theory, algorithms and applications," IEEE Trans. Compon., Packag., Manuf. Technol., 2018.

[36] B. Efron, T. Hastie, I. Johnstone, and R. Tibshirani, "Least angle regression," The Annals of statistics, vol. 32, no. 2, pp. 407-499, 2004.

[37] T. Nguyen, D. Mac, and S. Clénet, "Uncertainty quantification using sparse approximation for models with a high number of parameters: Application to a magnetoelectric sensor," IEEE Commun. Mag., vol. 52, no. 3, pp. 1-4, 2016

[38] W. Zhao, T. H. Beach, and Y. Rezgui, "Efficient least angle regression for identification of linear-in-the-parameters models," Proc. R. Soc. A, vol. 473 , no. 2198 , p. 20160775 , 2017.

[39] R. Trinchero, P. Manfredi, I. S. Stievano, and F. G. Canavero, "Machine learning for the performance assessment of high-speed links," IEEE Trans. Electromagn. Compat., 2018.

[40] M. Ahadi and S. Roy, "Sparse linear regression (spliner) approach for efficient multidimensional uncertainty quantification of high-speed circuits.," IEEE Trans. Comput.-Aided Design Integr. Circuits Syst., vol. 35, no. 10, pp. 1640-1652, 2016.

[41] R. Hu, V. Monebhurrun, R. Himeno, H. Yokota, and F. Costen, "An adaptive least angle regression method for uncertainty quantification in FDTD computation," IEEE Trans. Antennas Propag., vol. 66, no. 12, pp. 2131-2134, 2018.

[42] M. D. McKay, R. J. Beckman, and W. J. Conover, "Comparison of three methods for selecting values of input variables in the analysis of output from a computer code," Technometrics, vol. 21, no. 2, pp. 239-245, 1979.

[43] R. L. Iman, "Uncertainty and sensitivity analysis for computer modeling applications.," ASME AEROSP DIV PUBL AD., ASME, NEW YORK, NY(USA), 1992,, vol. 28, pp. 153-168, 1992.

[44] H. Hotelling, "Analysis of a complex of statistical variables into principal components.," Journal of educational psychology, vol. 24, no. 6, p. 417, 1933.

[45] I. T. Jolliffe, "Principal component analysis and factor analysis," in Principal component analysis, pp. 115-128, Springer, 1986.

[46] A. E. Hoerl and R. W. Kennard, "Ridge regression: Biased estimation for nonorthogonal problems," Technometrics, vol. 12, no. 1, pp. 55-67, 1970.

[47] P.-Y. Wu, C.-C. Fang, J. M. Chang, and S.-Y. Kung, "Cost-effective kernel ridge regression implementation for keystroke-based active authentication system," IEEE Trans. Cybern., vol. 47, no. 11, pp. 39163927, 2017.

[48] A. S. Goldberger, "Econometric theory," Econometric theory, 1964. 
[49] D. Hong, N. Yokoya, and X. X. Zhu, "Learning a robust local manifold representation for hyperspectral dimensionality reduction," IEEE J. Sel. Topics Appl. Earth Observ., vol. 10, no. 6, pp. 2960-2975, 2017.

[50] M. Mohanpurkar and S. Suryanarayanan, "Regression modeling for accommodating unscheduled flows in electric grids," IEEE Trans. Power Syst., vol. 29, no. 5, pp. 2569-2570, 2014.

[51] C. O. S. Sorzano, J. Vargas, and A. P. Montano, "A survey of dimensionality reduction techniques," arXiv preprint arXiv:1403.2877, 2014.

[52] J. S. Ochoa and A. C. Cangellaris, "Random-space dimensionality reduction for expedient yield estimation of passive microwave structures," IEEE Trans. Microw. Theory Tech., vol. 61, no. 12, pp. 4313-4321, 2013.

[53] A. Zare, A. Ozdemir, M. A. Iwen, and S. Aviyente, "Extension of pca to higher order data structures: An introduction to tensors, tensor decompositions, and tensor pca," Proceedings of the IEEE, vol. 106, no. 8, pp. 1341-1358, 2018.

[54] Q. Wang, Q. Gao, X. Gao, and F. Nie, "12, p-norm based pca for image recognition," IEEE Trans. Image Process., p. 1, 2016.

[55] N. Shahid, N. Perraudin, G. Puy, and P. Vandergheynst, "Compressive pca for low-rank matrices on graphs," IEEE transactions on Signal and Information Processing over Networks, vol. 3, no. 4, pp. 695-710, 2017.

[56] A. De Pierrefeu, T. Löfstedt, F. Hadj-Selem, M. Dubois, R. Jardri, T. Fovet, P. Ciuciu, V. Frouin, and E. Duchesnay, "Structured sparse principal components analysis with the TV-elastic net penalty," IEEE Trans. Med. Imag., vol. 37, no. 2, pp. 396-407, 2018.

[57] B. Schölkopf, A. Smola, and K.-R. Müller, "Nonlinear component analysis as a kernel eigenvalue problem," Neural computation, vol. 10, no. 5, pp. 1299-1319, 1998.

[58] M. A. Kramer, "Nonlinear principal component analysis using autoassociative neural networks," AIChE journal, vol. 37, no. 2, pp. 233-243, 1991.

[59] M.-C. Gosselin, A. Christ, S. Kühn, and N. Kuster, "Dependence of the occupational exposure to mobile phone base stations on the properties of the antenna and the human body," IEEE Trans. Electromagn. Compat., vol. 51, no. 2, pp. 227-235, 2009.

[60] M. Thapa, S. B. Mulani, and R. W. Walters, "A new non-intrusive polynomial chaos using higher order sensitivities," Computer Methods in Applied Mechanics and Engineering, vol. 328, pp. 594-611, 2018.

[61] J. Zeng, S. Lin, Y. Wang, and Z. Xu, " $L_{1 / 2}$ regularization: Convergence of iterative half thresholding algorithm," IEEE Trans. Signal Process., vol. 62, no. 9, pp. 2317-2329, 2014.

[62] W. T. Federer and B. T. Scully, "A parsimonious statistical design and breeding procedure for evaluating and selecting desirable characteristics over environments," Theoretical and Applied Genetics, vol. 86, no. 5, pp. 612-620, 1993.

[63] J.-P. Bérenger, "A perfectly matched layer for the absorption of electromagnetic waves," Journal of computational physics, vol. 114, no. 2, pp. 185-200, 1994.

[64] S. D. Gedney, G. Liu, J. A. Roden, and A. Zhu, "Perfectly matched layer media with CFS for an unconditionally stable ADI-FDTD method," IEEE Trans. Antennas Propag., vol. 49, no. 11, pp. 1554-1559, 2001.

[65] "The RIKEN webpage, media parameters for the Debye relaxation model," http://cfd-duo.riken.jp/cbms-mp/ Accessed: Sept. 3, 2018.

[66] R. Tibshirani, "Regression shrinkage and selection via the lasso," Journal of the Royal Statistical Society. Series B (Methodological), pp. 267288, 1996.

\section{APPENDIX A}

\section{MATHEMATICAL NOTATIONS}

$\boldsymbol{E} \quad$ Electric field

$\psi \quad$ Polynomial basis

$r \quad$ Order of NIPC expansion

$\mathcal{K}$ Number of Debye parameters of interest

$\xi \quad$ Debye parameters of interest

$b \quad$ Index of polynomial basis

$\boldsymbol{b} \quad$ Collective form of $b_{1} b_{2} \cdots b_{\mathcal{K}}$

$\mathcal{L}$ Number of polynomial bases in the NIPC expansion

$\mathcal{M}$ Number of samples

$\mathcal{X}$ Sample set

$\begin{array}{cl}\tilde{\mathcal{X}} & \text { Standardised } \mathcal{X} \\ \mathcal{G} & \text { Covariance matrix } \\ \mathcal{C} & \text { Vector of variance explained } \\ \widehat{\mathcal{C}} & \text { Cumulative summation of the elements of } \mathcal{C} \\ \mathcal{D} & \text { Number of remained Debye parameters } \\ \mathcal{X}_{\text {sub }} & \text { Transformed subset of } \widetilde{\mathcal{X}} \\ \mathcal{T}\left(\boldsymbol{\xi}^{(m)}\right) & \text { Predicted value of }|\boldsymbol{E}|^{2} \text { for } \boldsymbol{\xi}^{(m)} \\ a & \text { Coefficient of } \psi \\ \varepsilon_{l} & \text { LOO error } \\ v & \text { Penalty factor } \\ \mu & \text { Average value } \\ \mu(\mathcal{M}) & \text { Average value from the MCM } \\ \sigma(\mathcal{M}) & \text { Standard deviation from the MCM } \\ \sigma & \text { Standard deviation }\end{array}$

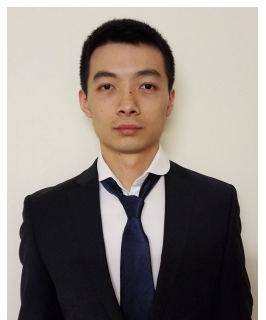

Runze Hu received the B.S. degree in computer science from the North China Electric Power University, Baoding, China, in 2014, and the M.Sc. degree in computer science from the University of Manchester, Manchester, U.K., in 2016. He started his Ph.D. study in Electrical and Electronics Engineering, University of Manchester, U.K., in 2017. His current research interests include artificial intelligence, uncertainty quantification techniques, and high-performance computing.

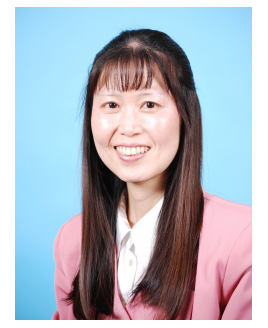

Fumie Costen (M'07) received the B.Sc. degree, the M.Sc. degree in electrical engineering and the Ph.D. degree in Informatics, all from Kyoto University, Japan. From 1993 to 1997 she was with Advanced Telecommunication Research International, Kyoto, where she was engaged in research on direction-of-arrival estimation based on Multiple SIgnal Classification algorithm for 3-D laser microvision. She filed three patents from the research in 1999 in Japan. She was invited to give 5 talks in Sweden and Japan during 1996-2014. From 1998 to 2000, she was with Manchester Computing in the University of Manchester, U.K., where she was engaged in research on metacomputing and has been a Lecturer since 2000 . Her research interests include computational electromagnetics in such topics as a variety of the finite difference time domain methods for microwave frequency range, microwave imaging, application of deep learning to FDTD, radar images and medical images. She filed a patent from the research on the boundary conditions in 2012 in the U.S.A. Her work extends to the hardware acceleration of the computation using general-purpose computing on graphics processing units, Streaming Single Instruction Multiple Data Extension and Advanced Vector eXtentions instructions. Dr. Costen received an ATR Excellence in Research Award in 1996 and a best paper award from 8th International Conference on High Performance Computing and Networking Europe in 2000.

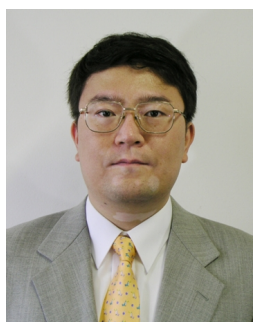

Hideo Yokota received his Doctor of Engineering degree from the University of Tokyo in 1999. In 1993, he joined Higuchi Ultimate Mechatronics Project, Kanagawa Academy of Science and Technology, Kawasaki, Japan. In 1999, he joined RIKEN and is the contract researcher of Computational biomechanics unit. 2004-2012 Bio-research Infrastructure Construction Team Leader, VCAD system research program, RIKEN. 2007-2012 Cell-scale Research and Development Team Leader,Research Program for Computational Science, RIKEN. 2013present Image Processing Research Team Leader, Center for Advanced Photonics,RIKEN. He is also a visiting Professor at Hokkaido University, Kobe University, Tokai University and the Tokyo University of Agriculture and Technology. He currently studies biomedical imaging and image processing to the biomedical simulation. Bioimaging Society, Best Image Award, 2005. The Commendation for Science and Technology by the Minister of Education, Culture, Sports, Science and Technology, Young Scientists Prize in 2008. 
Vikass Monebhurrun (SM'07) received the PhD degree in electronics in 1994 and the Habilitation à Diriger des Recherches (HDR) in physics in 2010 from Université Pierre et Marie Curie (Paris VI) and Université Paris-Sud (Paris XI), respectively. $\mathrm{He}$ was engaged in research on electromagnetic non-destructive testing for nuclear power and aeronautical applications until 1998, following which he joined the Department of Electromagnetics at Supélec (CentraleSupélec since 2015). His research interests encompass time domain numerical modeling as well as radio frequency measurements. He actively participated in French National Research Programs on electromagnetic dosimetry since 1998, namely COMOBIO (1999-2002 on 2G systems), ADONIS (20032005 on 3G systems) and MULTIPASS (2007-2010 on 4G systems). His research contributed to international standardization committees of CENELEC (European Committee for Electrotechnical Standardization), International Electrotechnical Commission (IEC), and IEEE. He is author and co-author of more than hundred peer-reviewed international conference and journal papers. He also holds three international patents on antennas for mobile communications. He is an active contributor to international standardization committees of IEC 62209, IEC 62232, IEC/IEEE 62704 and IEEE1528. He was a member of the European COST Action BM 1309. Dr. Monebhurrun serves as member of the Editorial Board of the IEEE COMPUMAG and IEEE CEFC conferences, and IEEE Transactions on Magnetics special issues since 1998. He is the founder of the IEEE Radio and Antenna Days of the Indian Ocean (RADIO) international conference and he served as General Chair for all seven editions since 2012. In 2013, he initiated the Radio Society (Mauritius) for which he serves as President. He is currently the Chair of the international committees of IEC/IEEE 62704-3 and IEEE Antennas and Propagation Standards. He further serves as member within several committees of the IEEE Antennas and Propagation Society. Since 2019, he is AdCom member of the IEEE Sensors Council and Corresponding Member of the Conference Coordination and Standards Coordination Subcommittees of IEEE Region 8. He serves as Associate-Editor for the IEEE Antennas and Propagation Transactions and Magazine, and Editor of the IoP Conference Series: Materials Science and Engineering. He was the recipient of the Union Radio-Scientifique Internationale (URSI) Young Scientist Award in 1996. Following the publication of the dual/logo IEC/IEEE 62704-3 international standard on computational dosimetry in 2017, he was awarded the IEEE Standards Association International Working Group Chair Award. As Chair of IEEE SIGHT Indian Ocean, he was presented the Ulrich L. Rohde Humanitarian Technical Field Project Award in 2018 for the Agriculture, Climate and Technology in Indian Ocean Network (ACTION) project. He was also the recipient of the IEC 1906 Award in 2018.

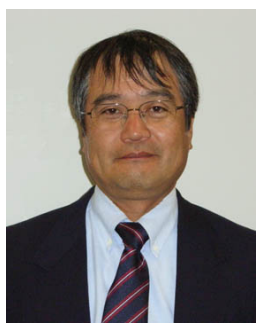

Ryutaro Himeno received his Doctor of Engineering degree from the University of Tokyo in 1988. In 1979, he joined Central Research Laboratories, Nissan Motor Co., Ltd., Yokosuka, Japan, where he has been engaged in the research of applying Computational Fluid Dynamics analysis to the car aerodynamic development. In 1998, he joined RIKEN and is a coordinator of R\&D Group, Head Office for Information Systems and Cybersecurity at RIKEN. He is also a visiting professor at Hokkaido University, Kobe University and Tokyo Denki University. He currently studies Computational Bioengineering, High Performance Computing and blood flows of human bodies. He was a winner of Nikkei Science, Computer Visualization Contest in 2000 and Scientific Visualization Contest in 1996, and received JSME Computational Mechanics Division Award in 1997 and JSME Youth Engineer Award in 1988. He has also received the Paper Award by NICOGRAPH in 1993, Giga FLOPS Award by CRAY Research Inc. in 1990 and other awards. 